\title{
Musculoskeletal and Performance Effects of Monocular Display Augmented, Articulated Arm Based Laser Digitizing
}

\author{
Neil Littell $^{1}$, Kari Babski-Reeves ${ }^{2}$, Gary McFadyen ${ }^{1}$, and John McGinley ${ }^{1}$ \\ ${ }^{1}$ Center for Advanced Vehicular Systems \\ Mississippi State University \\ 200 Research Blvd. \\ Mississippi State, MS 39762 \\ ${ }^{2}$ Department of Industrial and Systems Engineering \\ Mississippi State University \\ PO Box 9542 \\ Mississippi State, MS 39762
}

\begin{abstract}
Processes of capturing solid geometry features as three-dimensional data for analysis, simulation, or reverse engineering require the use of laserbased reverse engineering hardware, commonly known as digitizers. The most common digitizers used within manufacturing contexts are articulated armbased coordinate measuring machines, which have been augmented with a laser-head probe. Typical usage times for the digitizing equipment can range into the hours, thereby placing operators at risk for the development of musculoskeletal disorders (MSDs), though exact load magnitudes of exposure to risk factors for MSDs during object digitization are unknown. Further, other technologies (such as monocular/heads-up displays) may be combined with laser digitizers that may reduce load magnitudes. This paper explores the possibility of an occluded monocular display augmentation, results and discussion are presented.
\end{abstract}

Keywords: Monocular Display, Head Mounted Display, HMD, Augmented Reality Interface, Laser Digitizing.

\section{Introduction}

Processes of capturing solid geometry features as three-dimensional data for analysis, simulation, or reverse engineering require the use of laser-based reverse engineering hardware, commonly known as digitizers. The most common digitizers used within manufacturing contexts are articulated arm-based coordinate measuring machines, which have been augmented with a laser-head probe. Typical usage times for the digitizing equipment can range into the hours, thereby placing operators at risk for the development of musculoskeletal disorders (MSDs), though exact load magnitudes of exposure to risk factors for MSDs during object digitization are unknown. Further, other technologies (such as monocular/heads-up displays) may be combined with 
laser digitizers that may reduce load magnitudes. Additionally, operators must constantly monitor feedback from the system via a traditional computer monitor to ensure that certain conditions are being met and sufficient data are collected to construct the virtual part. Thus, the existing visual feedback system introduces additional rotation loads on the neck musculature. Further, other technologies (such as monocular/heads-up displays) may be combined with laser digitizers that may reduce load magnitudes.

Monocular displays are a potential technology that may be combined with laser digitizers to reduce injury risk as well as improve operator performance. Problems typically associated with using a monocular display include simulator sickness, binocular rivalry, and accommodation issues. All of these adverse side effects are caused cognitively, in that the user's brain cannot resolve the differences in what each eye is viewing. Peli (1990) noted that most of these problems can be overcome by positioning the monocular display in the lower region of the user's field of view; specifically, 15 degrees below the users line of sight (the bifocular region). To the users, the display appears to be in the same position as a piece of paper would if the user was holding it close to his or her chest, allowing the display to operate within the users peripheral, however it is not large enough to disrupt normal vision (Peli 1990).

Laser digitizing equipment performs satisfactorily, though this emerging technology forces the operator into non-neutral postures for prolonged periods of time, specifically for the upper extremities, back, and cervical spine. The incorporation of other commercially available technologies (such as monocular displays) may improve ergonomic exposures and operator performance. Therefore, the objectives of the study were to: (1) quantify musculoskeletal loads associated with articulated arm usage for the neck, shoulder, and back, and (2) quantify the impact of incorporating heads up (monocular) displays during object digitization on musculoskeletal loads and digitizing efficiency.

\section{Methods}

\subsection{Experimental Design}

A repeated measures design was used to test for the effects of task condition (2 levels) on muscle activity, posture, performance, and discomfort and usability perceptions. Participants performed a digitizing task using the FARO brand laser digitizer with and without the use of an augmented, monocular display. Participant exposure to the task conditions was counterbalanced.

\subsection{Independent Variable}

The independent variable for this study was task condition: with and without the use of the monocular display. A MicroOptical brand (SV-6 PC, 33 Southwest Park, Westwood, MA 02090) occluding monocular display was selected for use in this study. The display accepts a standard 800 X 600 resolution and outputs an image at $600 \times 480$ pixels. This display was chosen because literature suggest that using an 
occluding monocular display is not significantly different than using a traditional monitor for simple reference tasks (Peli, 1998; Laramee and Ware, 2002). Also, an occluded monocular display enabled users to place the image within their bifocular region. The current technology for transparent monocular displays offers limited freedom with this respect.

\subsection{Dependent Variables}

Four general dependent variables were considered: muscle activity, posture, performance, and discomfort and usability perceptions. Details for each of these are provided.

Muscle Activity. Muscle activity was collected using surface electromyography (EMG) during each test session using standard clinical procedures. EMG surface electrodes $(10 \mathrm{~mm}, \mathrm{Ag} / \mathrm{AgCl}$ pregelled bipolar electrodes) were attached in a bipolar arrangement over the sternocleidomastoid in the neck; the trapezius, levator scapule, and rhomboid major muscles of the shoulder blade region; and the multifidi-erector spinea complex muscles of the lower back of the dominate side of participants according to recommended procedures (Perotto, 1994). Preparatory procedures included shaving excess hair from the electrode attachment site, slightly abrading the skin with a polishing stone, and cleansing the area with alcohol. Electrodes were attached and following a 10-minute stabilization period impedance was checked (required to be less than $10 \mathrm{k} \Omega$ for each electrode pair) using a standard mulitmeter. Resting EMG signals were captured while the participant stood relaxed for a 6 second period. For the neck muscle, a submaximal exertion was performed by having participants bend $90^{\circ}$ from the waist (with the back and neck in alignment) while supporting a $3 \mathrm{lb}$ weight that was suspended from the head. For the shoulder muscles and back muscles, participants completed maximum voluntary contractions (MVCs). Participants grasped a height adjustable handle, stood erect with their arm resting at their side, and performed a shrugging motion with their shoulder to elicit shoulder muscle activity. For the low back, participants were seated in a backless chair with a strap around the chest attached to a wall, and performed an extension exertion (leaned backwards from the wall). For all MVCs and the neck submaximal exertion, three, five second exertions using a ramp-up, ramp-down procedure with a 30 second rest period between exertions were performed. The maximum value obtained for each muscle, regardless of MVC task was used as the MVC for that muscle. Task EMG data were collected continuously during digitizing. All data were sampled at $256 \mathrm{~Hz}$, pre-amplified, RMS converted, and filtered using a Butterworth filter (Noraxon Telemyo 2400R and MyoResearcher Software, 13430 N. Scottsdale Road, Suite 104 Scottsdale, Arizona 85254). Task EMG data were normalized before analysis. Again, the maximum value observed for each muscle will be used in data normalization. Mean percent of maximum was used to investigate muscle activity differences between task conditions.

Posture. A 12-camera motion capture system (Motion Analysis, EVaRT 4.6, 3636 N. Laughlin Road, Suite 110 Santa Rosa, CA 95403) was used to capture operators' motions. Participants wore a suit that allowed for the attachment of markers via 
Velcro. A standard full-body marker location protocol was used to facilitate analysis using ergonomics analysis software and methods. Motion data was collected at a rate of $60 \mathrm{~Hz}$.

Performance. Data collected by the subjects using the laser digitizer was analyzed using the companion software Geomagic, developed for post processing of digitized data. Specifically, the amount of missing data (holes) in terms of area and number of missing elements were used to determine differences between scanning sessions. Time to capture data was monitored and the number of bumps was recorded. While digitizing, it is important that the operator not bump the specimen, specimen support, or the digitizer itself. Doing so translates into a shift of the actual geometry of the specimen, thus creating a digital shift in pre vs. post disturbed geometry. Any disturbance classified as a bump will require the operator to start over at the beginning of the digitizing process. For this study, bumps were recorded, however the participants were instructed to continue digitizing as though the bump had not occurred.

Discomfort and Usability. Participants completed a body discomfort map at the end of each task condition, and completed a usability questionnaire at the end of the test session. The body discomfort map included a picture of the human body divided into regions (eg, head/neck, upper back, lower back, etc.) with associated visual analog scales (VAS) $10 \mathrm{~cm}$ in length with the anchors of "No discomfort" to "Extreme discomfort". Participants placed a vertical mark on each VAS scale to indicate the level of discomfort they were experiencing. Participants completed a usability questionnaire that contained questions pertaining to their perceptions of the digitizing equipment and the use of the monocular display. Participants rated a series of questions on a 5 point Likert-type scale ranging from $1=$ strongly disagree to $5=$ strongly agree.

\subsection{Task}

The task consisted of digitizing a small teapot using both task conditions. Participants continued digitizing until they indicated that they were finished. No feedback was provided to participants on performance during testing.

\subsection{Participants}

Ten participants ( 6 males and 4 females), ranging in age from 19 to 31 years completed study protocols. Participants were required to be free of arm, neck, and back injuries as evidenced by self report and have no history of motion sickness to be eligible to participate.

\subsection{Procedure}

Participants completed informed consent documents prior to data collection. EMG electrodes were applied and impedance checked prior to the attachment of the motion capture suit. Following motion capture calibration, a familiarization session was conducted in which participants practiced using a FARO brand articulated digitizer to 
scan a small object both with and without the use of the monocular display. Feedback on performance was provided. Participants then completed the two test conditions, were debriefed, and monetarily compensated for their time.

\subsection{Data Analysis}

Appropriate descriptive statistics were computed. A repeated measures ANOVA was performed to tests for differences between task conditions on the dependent variables. SAS 9.1 software was used for all analysis. As this was an exploratory study, an alpha level of 0.10 was used to determine significant findings.

\section{Results}

Table 1 presents the descriptive statistics for this study. In general, there is a trend for more favorable readings for the monocular display (i.e., mean muscle activity was lower for the monocular condition.

Table 1. Descriptive statistics. Values are mean (standard deviation).

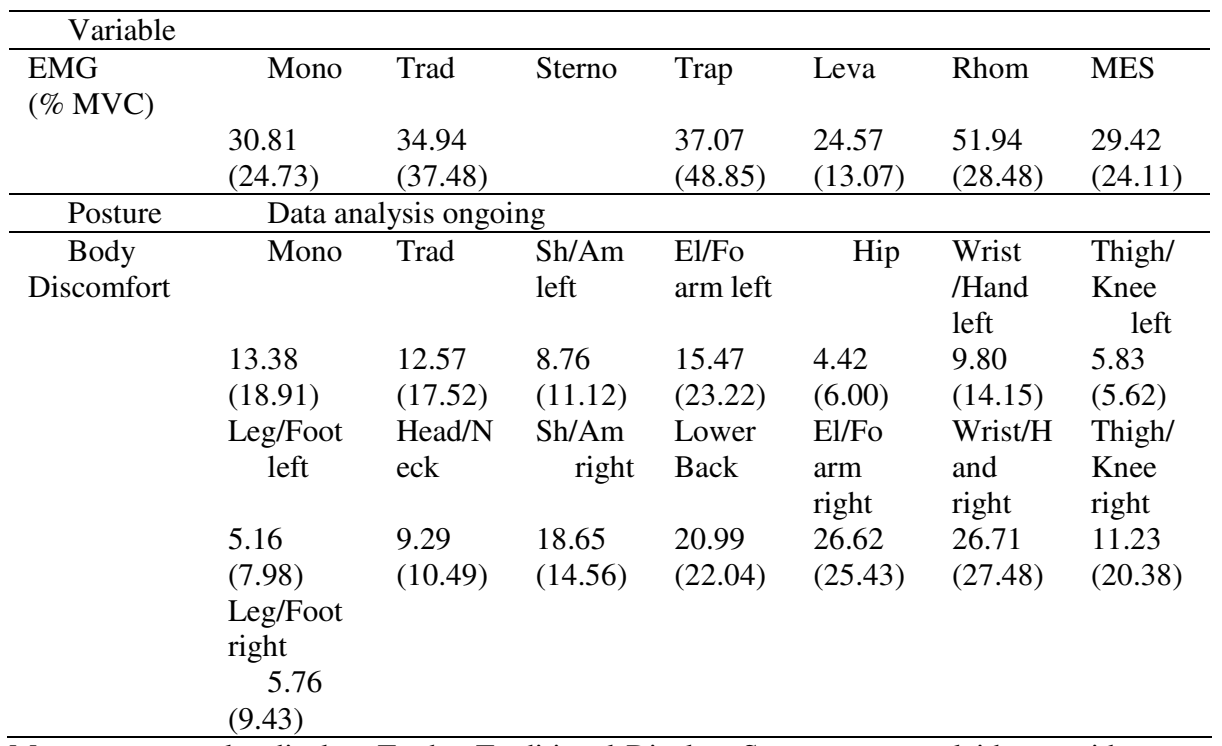

Mono $=$ monocular display, Trad $=$ Traditional Display, Sterno $=$ sternocleidomastoid, trap $=$ trapezius, Leva = levator scapule, Rhom $=$ rhomboid major, MES = multifidi-erector spinea complex.

\subsection{EMG and Posture}

No significant differences were found between the task conditions for any muscle investigated $(p=0.6253)$. There were differences found among the muscles $(p=0.943)$, with the rhomboid major being the most active and the sternocleidomastoid being the least active. At this time, posture data is unavailable. 


\subsection{Usability and Discomfort}

Discomfort ratings were not found to differ significantly across conditions or for any condition $(\mathrm{p}=0.6491)$ by body part interaction $(0.9355)$. There were significant differences across body parts, though this was expected. In general, lower back, elbow and forearm right, and wrist and hand right were found to have significantly higher ratings of discomfort than the other body parts.

\subsection{Performance}

The data collected by the participants was shown to be almost identical to one another. This is not surprising, because the particpants were instructed to digitize the models as completely as possible. Bumps were recorded, however there was not enough data collected to be analyzed in any meaningful way.

\subsection{Discomfort and Usability}

Usability ratings are presented in figure 1 below. Six questions were found to have significantly higher usability ratings. Four of these questions asked participants if they preferred the monocular display over the traditional laptop, or that the faro arm was more comfortable to use with the monocular display. The remaining two questions asked about improved performance with additional practice for the monocular and traditional display, and both received ratings well over 4 (agree). Two additional

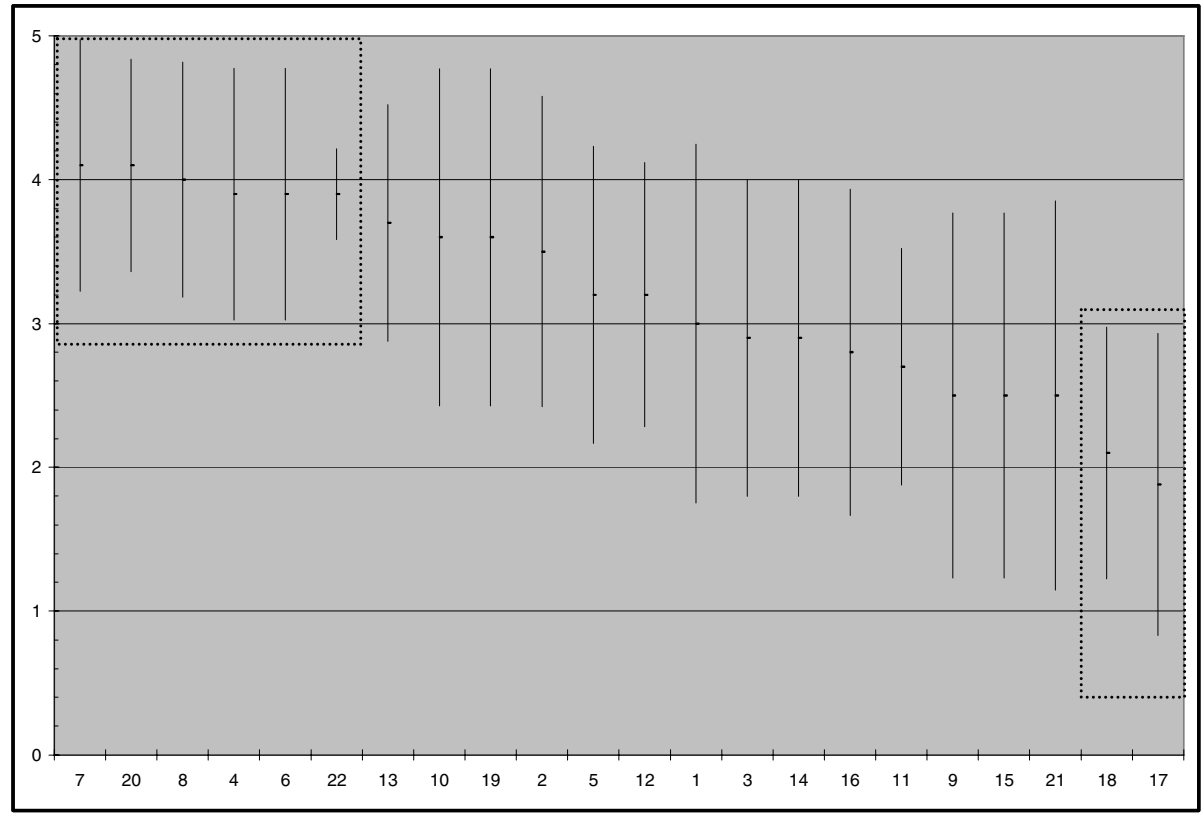

Fig. 1. Participants Responses to Survey 
questions received significantly lower responses than the others. These two questions, which had average ratings near 2 (disagree) asked participants if they would be comfortable scanning with the FARO arm for 8 hours a day.

\section{Discussion and Conclusions}

The objectives of this study were to quantify ergonomic exposures associated with laser digitizer use and to assess if the use of an augmented monocular display would improve exposures and performance. Results of this study indicated that significant ergonomic loads are placed on the shoulder, neck, and back musculature. In general, muscle activity levels were near 25\% MVC or greater. Studies have found localized muscle fatigue to occur at low levels of muscle activity, particularly when the tasks are static in nature (e.g., Veiersted et al, 1993; Forsman et al, 2001). Further, these levels of activity were associated with relatively static postures and positions. Subscribing to the Cinderella hypothesis (Hägg, 1991), damage to the muscle fibers is likely given this task. Further data processing of the EMG data will investigate localized muscle fatigue rates associated with this job task. These findings are supported by the discomfort findings.

Discomfort results indicate that simply using the FARO arm, regardless of display type, results in significant levels of discomfort. Changes in the FARO arm display are needed based on anecdotal participant comments relating to the difficulty in using and supporting the digitizer during task performance.

In general there is some support for the use of an augmented display to improve user perceptions and ergonomic exposures. The sample size for this study however is insufficient to detect differences between the task conditions with sufficient power. Perhaps if experts, rather than novices, had been investigated differing results may have been found. A more comprehensive study is planned to further evaluate the ergonomic and usability effects of augmenting laser digitizers. This type of monocular display seems very appropriate for this type of task as it requires:

1. Frequent to real-time referencing of a visual feed back device.

2. Hands-on application.

3. Environment awareness (in this case, avoidance of the specimen/Faro arm).

4. Physical mobility.

Ultimately, the findings of this study were mixed, with some indication that augmentation to laser digitizers provides benefits to users. However, further studies are needed to explore these findings.

\section{References}

1. Forsman, M., Birch, L., Zhang, Q., Kadefors, R.: Motor-unit recruitment in the trapezius muscle with special reference to coarse arm movements. Journal of Electromyography and Kinesiology 11, 207-216 (2001)

2. Hägg, G.M: Static work loads and occupational myalgia-a new explanation model. In: Anderson, P.A., Hobart, D.J., Danoff, J.V. (eds.) Electromyographical Kinesiology, pp. 141-143. Elsevier Science, Amsterdam (1991) 
3. Laramee, R., Ware, C.: Rivalry and Interference with a head mounted display. ACM Transactions on Computer-Human Interaction 9(3), 238-251 (2002)

4. Peli, E.: Visual issues in the use of a head-mounted monocular display. Optical Engineering 29(8), 883-892 (1990)

5. Peli, E.: The Visual effects of head-mounted display (HMD) are not distinguishable from those of desk-top computer display. Vision Res. 38, 2053-2066 (1998)

6. Perotto, D.: Anatomical Guide for the Electromyographer: The Limbs and Trunk (3rd edn.), Charles C. Thomas, Springfield, IL (1994)

7. Veiersted, K.B., Westgaard, R.H., Andersen, P.: Electromyographical evaluation of muscular work pattern as a predictor of trapezius myalgia. Scandinavian Journal of Work, Environment and Health 19, 284-290 (1993) 